

\title{
Fundamental Benefits of the Staggered Geometry for Organic Field-Effect Transistors
}

\author{
Chang Hyun Kim, Yvan Bonnassieux, Gilles Horowitz
}

\section{To cite this version:}

Chang Hyun Kim, Yvan Bonnassieux, Gilles Horowitz. Fundamental Benefits of the Staggered Geometry for Organic Field-Effect Transistors. IEEE Electron Device Letters, 2011, 32 (9), pp.1302. 10.1109/LED.2011.2160249 . hal-00630469v2

\section{HAL Id: hal-00630469 \\ https://hal.science/hal-00630469v2}

Submitted on 29 Aug 2013

HAL is a multi-disciplinary open access archive for the deposit and dissemination of scientific research documents, whether they are published or not. The documents may come from teaching and research institutions in France or abroad, or from public or private research centers.
L'archive ouverte pluridisciplinaire HAL, est destinée au dépôt et à la diffusion de documents scientifiques de niveau recherche, publiés ou non, émanant des établissements d'enseignement et de recherche français ou étrangers, des laboratoires publics ou privés. 


\title{
Fundamental Benefits of the Staggered Geometry for Organic Field-Effect Transistors
}

\author{
Chang Hyun Kim, Yvan Bonnassieux, and Gilles Horowitz
}

\begin{abstract}
In this letter, decisive advantages of the staggered-type organic field-effect transistors (OFETs) over the coplanar-type are elucidated by two-dimensional device simulation. It is found that the charge transport in the channel is not limited by the contact electrode in staggered OFETs, whereas coplanar OFETs show strongly contact-limited behavior. This dissimilarity originates from the continuity (staggered) or discontinuity (coplanar) of the carrier concentration at the channel ends, which is directly connected to the channel potential profile. Calculated current-voltage curves also support these arguments as the current in coplanar OFETs follows the contact-limited transistor model.
\end{abstract}

Index Terms-Contact resistance, device geometry, organic field-effect transistors (OFETs), two-dimensional simulation

\section{INTRODUCTION}

$\mathrm{O}$ RGANIC field-effect transistors (OFETs) are under consistent development as a future candidate for low-cost flexible circuitry and display application. In spite of the impressive progress in their performance, there is still lack of relevant physical description or model that can adequately interpret (or predict) the observed phenomena and this is regarded as a current bottleneck for future developments.

The importance of the device geometry of OFETs has been often emphasized. Reference [1] gives the definition of the two basic categories; coplanar and staggered configurations. As most OFETs adopt the bottom-gate (BG) structure, the comparison of these two configurations reduces to that of the (source/drain) bottom-contact $(\mathrm{BC})$ and the top-contact (TC) structure. It is generally believed that TC devices have lower contact resistance $\left(R_{c}\right)$ [2] and suggested reasons comprise the morphological continuity of the semiconductor [2], [3] and/or the penetration of deposited metal clusters [4] that can favor the injection of carriers into the channel. However, these explanations are not supported by sufficient physical backgrounds.

In this study, we report on a purely physical reasoning on the beneficial effects of staggered-type OFETs by means of two-dimensional device simulation. We could rule out any morphological or process-related factors because the semiconductor is considered as a continuous medium and all interfaces are perfectly abrupt in the simulation. We found that the advantageous contact feature of staggered-type OFETs stems from the continuous carrier concentration along the conducting channel. The potential profiles and the current-voltage (I- $V$ ) characteristics confirmed this argument.

\section{Simulation Setup}

Comparative studies were conducted through physically-based two-dimensional device simulation (ATLAS simulator by SILVACO). This finite-element simulation involves solving a set of coupled Poisson's, continuity, and drift-diffusion equations within a user-defined two-dimensional structure.

C. H. Kim, Y. Bonnassieux, and G. Horowitz are with LPICM, Ecole Polytechnique, CNRS, 91128 Palaiseau Cedex, France (e-mail: chang-hyun.kim@polytechnique.edu; yvan.bonnassieux@polytechnique.edu; gilles.horowitz@polytechnique.edu). 
TABLE I

LIST OF THE SETUP PARAMETERS FOR ATLAS SIMULATION

\begin{tabular}{|c|c|c|}
\hline Material (layer) & Parameter & Value \\
\hline \multirow{6}{*}{$\begin{array}{c}\text { Pentacene } \\
\text { (semiconductor) }\end{array}$} & Ionization potential & $5.2 \mathrm{eV}$ \\
\hline & Electron affinity & $2.8 \mathrm{eV}$ \\
\hline & $\begin{array}{c}\text { Effective density of states at } \\
\text { the HOMO edge }\end{array}$ & $10^{20} \mathrm{~cm}^{-3}$ \\
\hline & P-type doping concentration & $0 \mathrm{~cm}^{-3}$ (undoped) \\
\hline & Dielectric constant & 3.6 \\
\hline & Hole mobility & $0.5 \mathrm{~cm}^{2} / \mathrm{V} \cdot \mathrm{s}$ \\
\hline \multicolumn{3}{|l|}{ (gate insulator) } \\
\hline $\begin{array}{c}\mathrm{Au} \\
\text { (source/drain) }\end{array}$ & Work function & $4.9 \mathrm{eV}(4.88 \mathrm{eV})^{\mathrm{a}}$ \\
\hline Al (gate) & Work function & $4.2 \mathrm{eV}$ \\
\hline
\end{tabular}

\footnotetext{
${ }^{\mathrm{a}} \mathrm{Au}$ work function is set to be $4.9 \mathrm{eV}$ for all simulations. $4.88 \mathrm{eV}$ is only additionally used in the calculations for the transfer curves with different injection barrier heights (Fig. 4).
}

Fig. 1 shows the structures of simulated OFETs based on the BG configuration. One hundred nanometers of pentacene was defined as the organic semiconductor and $300 \mathrm{~nm}$ of $\mathrm{SiO}_{2}$ for the gate insulator. The sole difference between Fig. 1(a) (BC) and Fig. 1(b) (TC) is the position of the source/drain electrodes. The origin of the xy coordinates is located at the starting point of the channel at the source side.

Table I lists the setup parameters used for the numerical simulation. The data library of ATLAS contains Pentacene model as a pre-defined material. Pentacene was assumed to be undoped based on our recent result in [5] and the dielectric constant was also taken from [5]. All other parameters including energy levels of pentacene and contact metals are common literature values as these materials have been widely investigated. Based on a recent comprehensive study of traps in pentacene [6], we also compare trap-free pentacene (which would correspond to pentacene single-crystals) to pentacene with an exponential distribution of traps (which rather describes polycrystalline pentacene film). Total trap density $\left(N_{t}\right)$ of $10^{18} \mathrm{~cm}^{-3}$ and trap characteristic temperature $\left(T_{c}\right)$ of $600 \mathrm{~K}$ are taken as the trap parameters.

\section{RESUlTS AND DISCUSSION}

Fig. 2 shows the potential profiles in both structures at a gate voltage $\left(V_{G}\right)$ of $-20 \mathrm{~V}$ and drain voltage $\left(V_{D}\right)$ of $-2 \mathrm{~V}$ (linear-regime). In BC OFETs (Figs. 2(a) and 2(b)), the potential at the channel $(\mathrm{y}=0.001 \mu \mathrm{m})$ is very close to that at the top surface $(y=0.099 \mu \mathrm{m})$, which validates the assumption in [7] for the potentiometry by scanning Kelvin probe microscopy (SKPM). By contrast, the channel potential in TC OFETs (Figs. 2(c) and 2(d)) does not coincide with the surface potential, which can be attributed to the gate voltage drop across the semiconductor layer (along the $y$ axis). Even though the trapped (fixed) charges can be expected to influence the electrostatic distribution, the effect of traps is marginal in the potential profiles. 

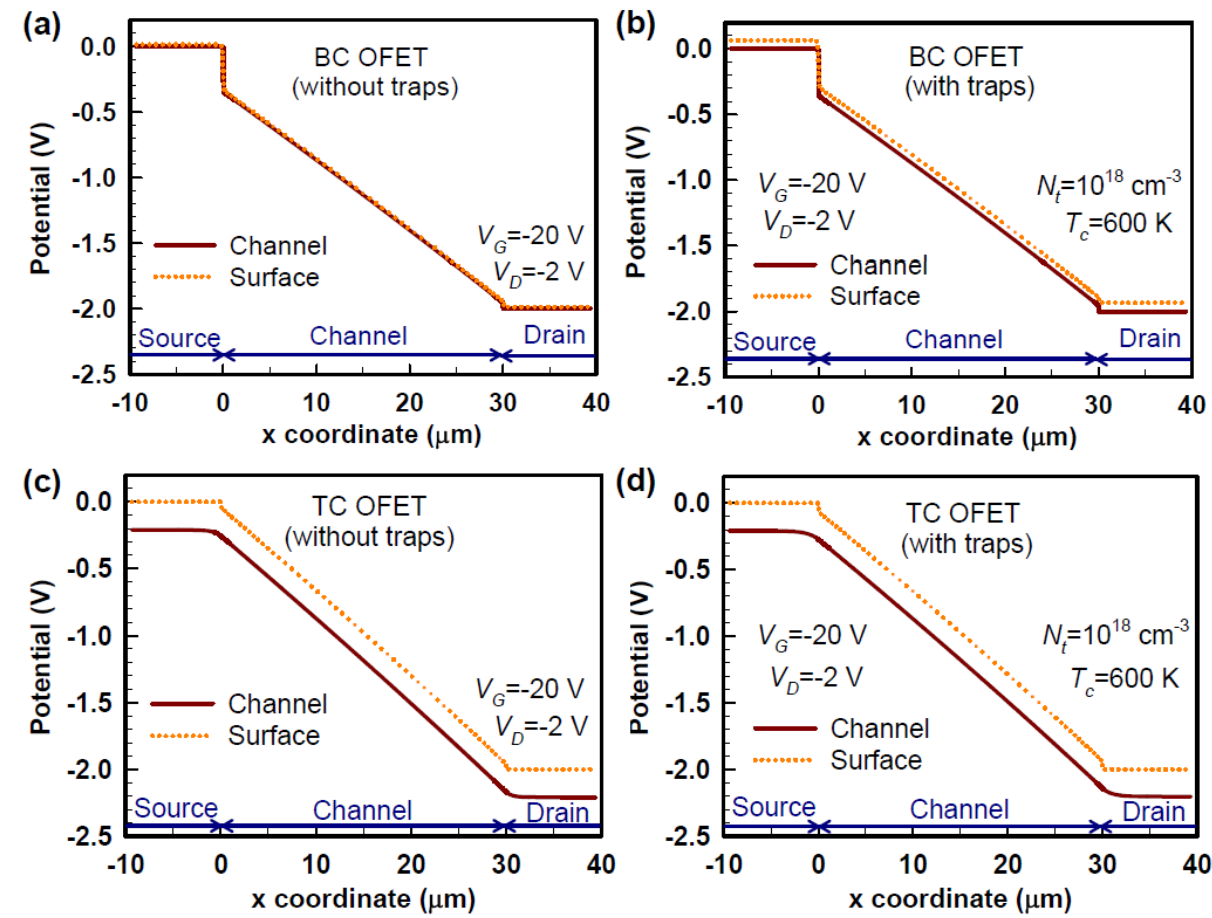

Fig. 2. Simulated potential profiles. (a) BC OFET without traps, (b) BC OFET with traps, (c) TC OFET without traps, and (d) TC OFET with traps. Upon the assumption of the equipotential channel at $V_{D}=0 \mathrm{~V}$, all extracted potential profiles are corrected with the reference profile at $V_{D}=0$ V.

The salient feature in Fig. 2 is the abrupt potential drop at the source/channel edge $(x=0)$ in BC OFET, which does not exist in TC OFET. This is consistent with the SKPM data in [2]. This potential drop is the root of high contact resistance $R_{c}$ in BC OFETs. In TC OFETs, the bulk resistance manifests itself by a voltage drop in the y direction [8], [9]. However, as will be seen in Fig. 4, the bulk resistance is negligible compared to the channel resistance because $V_{D}$ fully applies across the channel, so the effective injection length [8] is very small (checked by current mapping).

To find out the origin of the potential drop at the source electrode, we examine the (free) carrier distribution in both OFETs (Fig. 3). For a proper interpretation, one should keep in mind that the carrier density at the metal/semiconductor interface is dictated by Boltzmann statistics [10] so that the hole concentration at the source and drain electrodes $\left(p_{s}\right.$ and $\left.p_{d}\right)$ is given by:

$$
p_{s}=p_{d}=N_{V} \exp \left(-E_{b} / k T\right)
$$

which is independent of $V_{G}$ and $V_{D}$. Here, $N_{V}$ is the HOMO effective density of states, $E_{b}$ the (hole) injection barrier height from the source (or drain) into the HOMO of pentacene, $k$ the Boltzmann constant, and $T$ the absolute temperature. With $E_{b}=0.3 \mathrm{eV}$ (Table I), $p_{s}=p_{d}=9.7 \times 10^{14} \mathrm{~cm}^{-3}$.

By contrast, the hole concentration along the semiconductor/insulator interface $\left(p_{c h}\right)$ is induced by capacitive effect, and its dependence with $V_{G}$ writes:

$$
p_{c h}=Q_{c h} / q t_{c h}=C_{i}\left|V_{G}-V_{T}\right| / q t_{c h}
$$



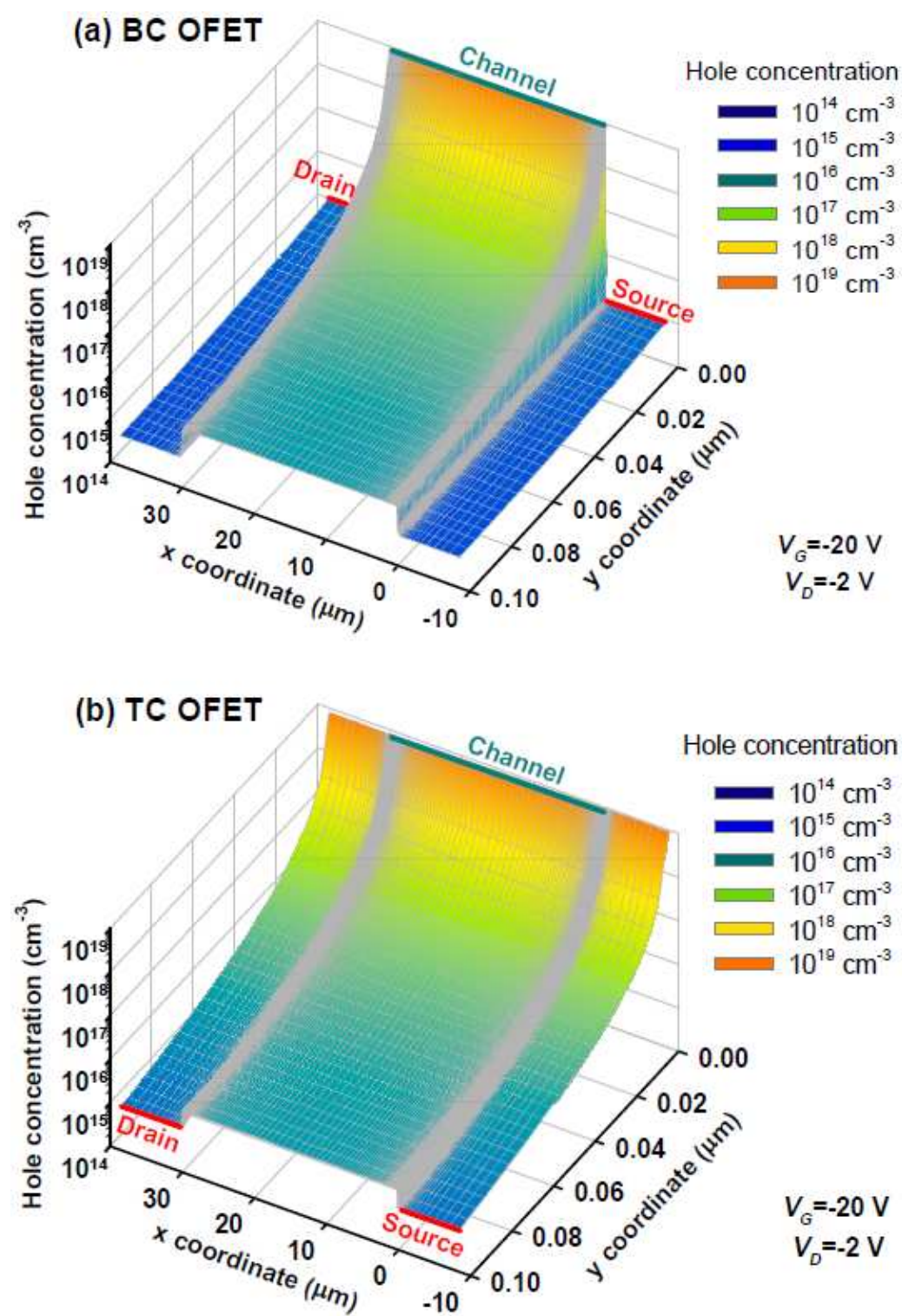

Fig. 3. 3D mesh plots showing the variation of the hole concentration in the semiconductor xy plane (trap-free case); (a) BC OFET and (b) TC OFET.

where $Q_{c h}$ is the channel charge density per unit area, $q$ the elementary charge, $t_{c h}$ the effective channel thickness, $C_{i}$ the gate capacitance per unit area, and $V_{T}$ the threshold voltage. The simulation (Fig. 3) gives $p_{c h}=8.0 \times 10^{18} \mathrm{~cm}^{-3}$ and we can calculate $t_{c h}$ by (2) (with $V_{T}$ estimated in Fig. 4). The result is $1.6 \mathrm{~nm}$, in accordance with other theoretical estimations [11].

Fig. 3(a) markedly indicates that in BC OFETs, the holes must be injected into the channel through a narrow transition zone where the hole density abruptly increases from $p_{s}$ to $p_{c h}$. As the (local) conductivity is proportional to the carrier density, and because $p_{s}$ is orders of magnitude lower than $p_{c h}$, this transition zone is highly resistive and gives rise to a high-resistance region $\left(R_{c}\right)$ at the source contact. The situation is totally different in TC OFETs (Fig. $3(b))$. Here, the semiconductor is always in contact with the insulator, so there is a nearly perfect continuity of the hole concentration at both ends of the channel $(\mathrm{x}=0$ and $\mathrm{x}=30 \mu \mathrm{m})$. This is coherent with the channel potential profile in Fig. 2(b) without any visible $R_{c}$ component in TC OFET. With traps, the overall hole density is slightly reduced due to the trapped charges but the same contrast between two structures is observed. 

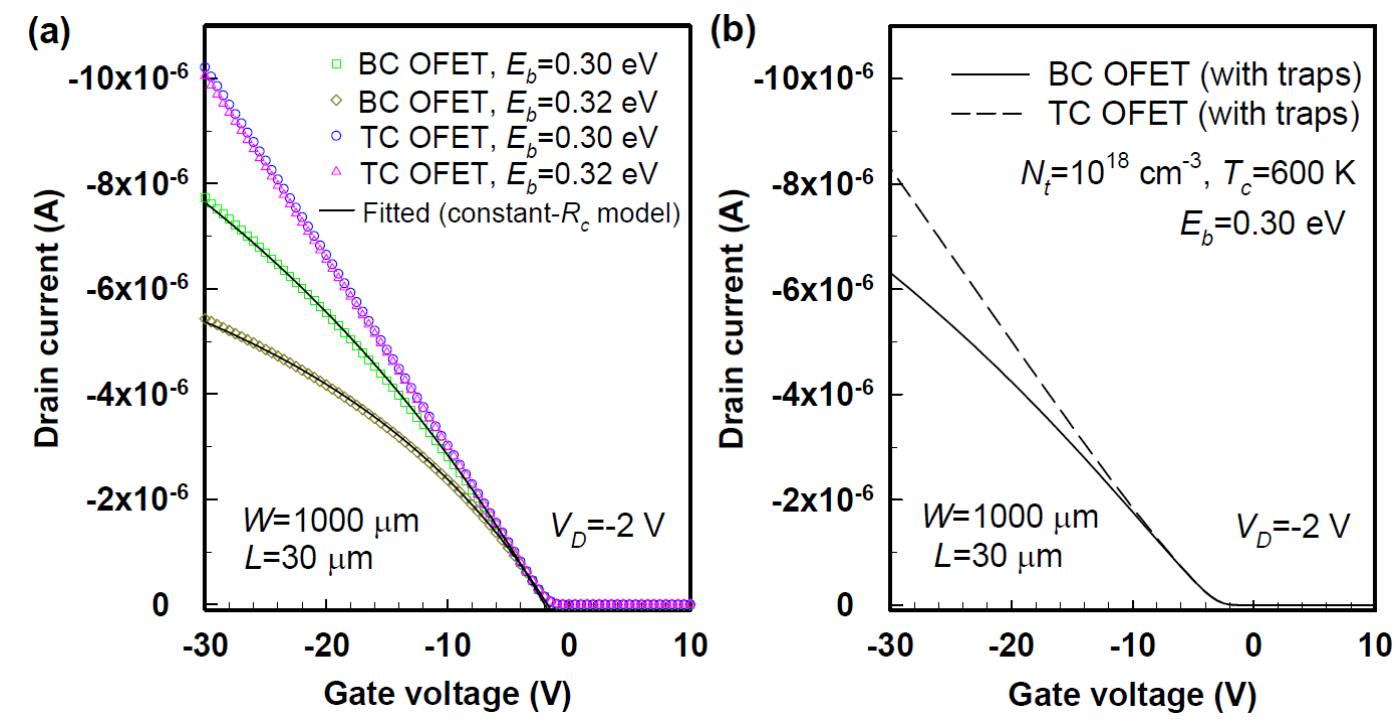

Fig. 4. (a) Simulated transfer characteristics of the trap-free BC and TC OFETs with two different injection barrier heights, (b) simulated transfer curves with exponential traps.

Calculated $I-V$ curves (transfer characteristics) in Fig. 4 show that this microscopic picture is in good agreement with the electrical performance of the OFETs. In Fig. 4(a), two different $E_{b}$ values $(0.30 \mathrm{eV}$ and $0.32 \mathrm{eV})$ were used to further accentuate the contrast between BC and TC OFETs; TC OFETs follow the ideal (contact-free) $I-V$ model where the drain current $\left(I_{D}\right)$ is linearly modulated by $V_{G}$ and $E_{b}$ does not play any critical role. On the contrary, $I_{D}$ of BC OFETs is strongly contact-limited, as indicated by the downward bending of the transfer curves. A slight increase $(0.02 \mathrm{eV})$ of $E_{b}$ results in a considerable decrease of the current. The shape of $\mathrm{BC}$ transfer curves could be well fitted to the constant $-R_{c}$ model in [12]. The extracted $R_{c}$ are $8.5 \times 10^{4} \Omega$ with $E_{b}=0.30 \mathrm{eV}$ and $1.8 \times 10^{5} \Omega$ with $E_{b}=0.32 \mathrm{eV}$. In comparison to Fig. 4(a), Fig. 4(b) indicates that introducing traps results in a significant decrease of the field-effect mobility and negative shift of the threshold voltage in both OFETs; traps reduce the density of free charge carriers and more negative $V_{G}$ is needed to induce a conductive channel [12]. Note that the TC architecture keeps its advantage over BC OFET even with traps because the channel hole distribution remains continuous.

\section{CONCLUSION}

Two-dimensional simulations of the coplanar and staggered OFETs were presented. The staggered geometry has obvious benefits for charge injection owing to a continuous carrier concentration in the whole channel area. This finding strongly motivates the adoption of the staggered geometry to achieve high-performance transistors with low parasitic resistance.

\section{REFERENCES}

[1] T. Richards and H. Sirringhaus, "Bias-stress induced contact and channel degradation in staggered and coplanar organic field-effect transistors," Appl. Phys. Lett., vol. 92, no. 2, pp. 023512-1-023512-3, Jan. 2008.

[2] K. P. Puntambekar, P. V. Pesavento, C. D. Frisbie, "Surface potential profiling and contact resistance measurements on operating pentacene thin-film transistors by Kelvin probe force microscopy," Appl. Phys. Lett., vol. 83, no. 26 pp. 5539-5541, Nov. 2003.

[3] C. Bock, D. V. Pham, U. Kunze, D. Käfer, G. Witte, Ch. Wöll, "Improved morphology and charge carrier injection in pentacene field-effect transistors with thiol-treated electrodes," J. Appl. Phys., vol. 100, no. 11, pp. 114517-1-114517-7, Dec. 2006.

[4] P. V. Pesavento, R. J. Chesterfield, C. R. Newman, C. D. Frisbie, "Gated four-probe measurements on pentacene thin-film transistors: Contact resistance as a function of gate voltage and temperature," J. Appl. Phys., vol. 96, no. 12, pp. 7312-7324, Aug. 2004.

[5] C. H. Kim, O. Yaghmazadeh, D. Tondelier, Y. B. Jeong, Y. Bonnassieux, G. Horowitz, "Capacitive behavior of pentacene-based diodes: Quasistatic dielectric constant and dielectric strength," J. Appl. Phys., vol. 109, no. 8, pp. 083710-1-083710-9, Apr. 2011.

[6] W. L. Kalb and B. Batlogg, "Calculating the trap density of states in organic field-effect transistors from experiment: A comparison of different methods," Phys. Rev. B, vol. 81, no. 3, pp. 035327-1-035327-12, Jan. 2010. 
[7] L. Bürgi, H. Sirringhaus, R. H.Friend, "Noncontact potentiometry of polymer field-effect transistors," Appl. Phys. Lett., vol. 80, no. 16, pp. 2913-2915, Feb. 2002.

[8] T. J. Richards and H. Sirringhaus, "Analysis of the contact resistance in staggered, top-gate organic field-effect transistors," J. Appl. Phys., vol. 102, no. 9, pp. 094510-1-094510-6, Nov. 2007.

[9] S. D. Wang, Y. Yan, K. Tsukagoshi, "Understanding contact behavior in organic thin film transistors," Appl. Phys. Lett., vol. 97, no. 6, pp. 063307-1-063307-3, Aug. 2010.

[10] R. S. Muller and T. I. Kamins, Device Electronics for Integrated Circuits. New York: Wiley, 2003, ch. 3.

[11] G. Horowitz, "Tunneling current in polycrystalline organic thin-film transistors," Adv. Funct. Mater., vol. 13, no. 1, pp. 53-60, Jan. 2003.

[12] D. Braga and G. Horowitz, "Subthreshold regime in rubrene single-crystal organic transistors," Appl. Phys. A-Mater. Sci. Process., vol. 95, no. 1, pp. 193-201, Dec. 2009. 